\title{
Transferrina como marcador de maturação pulmonar em cordeiros nascidos a termo ou prematuros
}

Rafaela Speranza Baptista“,Fernanda Bovino, Daniela Scantamburlo Denadai, Natalia Machado Rahal, Lilian Utraga dos Santos, Isabela Ferreira Peverari, Flavia de Almeida Lucas, Francisco Leydson Formiga Feitosa, Juliana Regina Peiró, Luiz Claudio Nogueira Mendes

Faculdade de Medicina Veterinária, Universidade Estadual Paulista (UNESP), Araçatuba, SP, Brasil

*Autor correspondente

e-mail: rafa.baptista@terra.com.br

\section{Resumo}

A prematuridade está associada à imaturidade de diversos órgãos, o que pode acarretar problemas no desenvolvimento do sistema respiratório, no metabolismo e enfermidades infecciosas. 0 objetivo do estudo foi procurar proteínas de fase aguda que possam indicar sinais de maturação no neonato prematuro através da quantificação sérica das mesmas. Identificou-se a imunoglobulina A, ceruloplasmina, haptoglobina, glicoproteína ácida, transferrina, albumina e as imunoglobulinas G de cadeias leve e pesada pela comparação do perfil dos proteinogramas de cordeiros nascidos a termo com os prematuros submetidos a diferentes protocolos terapêuticos, para estimular a atividade respiratória. Constituíram-se seis grupos: PN (n = 9): cordeiros nascidos de parto normal; $\mathrm{CN}(\mathrm{n}=7)$ : cordeiros nascidos de cesariana em tempo normal de gestação; $C P(n=6)$ : cordeiros nascidos de cesariana prematura sem nenhum tipo de tratamento; $D E X(n=9)$ cordeiros prematuros cujas mães receberam dexametasona pré-parto; SURF $(n=6)$ cordeiros prematuros tratados com surfactante; DEXSURF $(n=6)$ : cordeiros prematuros tratados com surfactante cujas mães receberam dexametasona pré-parto. As avaliações foram realizadas nos momentos imediatamente após o nascimento $\left(\mathrm{M}_{0}\right)$ e após 24 e 48 horas $\left(\mathrm{M}_{24}\right.$ e $\left.\mathrm{M}_{48}\right)$. As amostras foram processadas por meio de eletroforese em gel de poliacrilamida, contendo dodecil sulfato de sódio (SDS-PAGE), e os géis foram posteriormente avaliados pelo software ImageQuantTM TL. Antes da ingestão do colostro, as concentrações séricas de proteína total (Pt) foram maiores ao nascimento de $\mathrm{PN}$ em relação àqueles nascidos de $\mathrm{CN}$, e após a ingestão, as concentrações de Pt aumentaram nos momentos seguintes em relação aos valores do grupo a termo. Em relação às concentrações séricas proteicas, não houve diferença estatística entre os grupos nos animais nascidos de cesariana, apenas entre os momentos, cujo $\mathrm{M}_{0}$ apresentou menores valores séricos da Pt em todos os animais, elevando-se nos momentos seguintes, demonstrando que independente do tratamento, todos foram capazes de absorver as proteínas colostrais. Os maiores valores de transferrina no $\mathrm{M}_{0}$ foram 
descritos no grupo $\mathrm{CN}$ e os menores no grupo DEX. Nos momentos seguintes $\left(\mathrm{M}_{24}\right.$ e $\left.\mathrm{M}_{48}\right)$, os maiores valores foram também do grupo $\mathrm{CN}$, sugerindo que esta proteína pode indicar maturidade nos cordeiros, visto que os maiores valores encontrados são em animais a termo, quando comparados aos prematuros com os diversos tipos de tratamento. 0 grupo DEX foi o único que apresentou diferença estatística entre os momentos avaliados no estudo, onde apesar de apresentar os mais baixos valores séricos de transferrina ao nascer, apresentou às 48 horas as maiores médias dentre os prematuros, concluindo-se que o uso da dexametasona ajuda na maturação pulmonar de cordeiros. Em suma, as imunoglobulinas e a proteína total dos cordeiros tiveram elevação, independentemente dos grupos, após a ingestão de colostro. Maiores valores séricos de transferrina são referentes ao maior período gestacional, podendo esta proteína ser utilizada como marcador de maturação neonatal. 